\title{
BMJ Open Peer influence in adolescent drinking behaviour: a protocol for systematic review and meta-analysis of stochastic actor-based modeling studies
}

\author{
Valeria Ivaniushina, Vera Titkova, Daniel Alexandrov
}

To cite: Ivaniushina V, Titkova V, Alexandrov D. Peer influence in adolescent drinking behaviour: a protocol for systematic review and meta-analysis of stochastic actor-based modeling studies. BMJ Open 2019;9:e028709. doi:10.1136/ bmjopen-2018-028709

- Prepublication history and additional material for this paper are available online. To view please visit the journal (http:// dx.doi.org/10.1136/bmjopen2018-028709).

Received 21 February 2019

Revised 14 June 2019

Accepted 28 June 2019

\section{Check for updates}

(c) Author(s) (or their employer(s)) 2019. Re-use permitted under CC BY-NC. No commercial re-use. See rights and permissions. Published by BMJ.

Department of Sociology, National Research University Higher School of Economics, St. Petersburg, Russian Federation

Correspondence to Vera Titkova; vtitkova@hse.ru

\section{ABSTRACT}

Introduction Alcohol consumption is a considerable public health problem that is especially harmful to young people. To develop effective prevention programmes targeted at adolescents, it is important to understand the social mechanisms triggering alcohol consumption. Among such mechanisms, peer influence plays an important role. The effects of peer influence are very difficult to evaluate because of the entanglement with social selection, that is, a tendency of people to befriend others with similar behaviour. The recently developed stochastic actor-oriented models (SAOM) approach is designed to disentangle social influence from social selection. The aim of this study is to conduct a systematic review and meta-analysis of studies employing SAOM methodology to evaluate the effects of social influence on adolescent drinking behaviour.

Methods and analysis In order to analyse the coevolution of alcohol consumption and adolescent friendship networks, we will collect articles that use SAOM methodology through systematic electronic searches in Web of Science, Scopus, PubMed, The Cochrane Library (Cochrane Database of Systematic Reviews, Cochrane Central Register of Controlled Trials), EBSCOhost (MEDLINE, SocINDEX, Academic Source, ERIC), ProQuest (ProQuest Dissertations and Theses Global), PsycINF0 (PsycNET), Excerpta Medica database (Embase) and Cumulative Index to Nursing and Allied Health Literature (CINAHL). We will collect the literature from academic journals, dissertations/ theses, reports and conference materials. Three reviewers will retrieve and independently assess potentially relevant material in terms of whether they comply with prespecified criteria. Subsequently, we will summarise the results of the studies in a systematic review. If a sufficient number of studies can be found, SAOM quantitative results will be extracted and meta-analysed. The project will go from 1 December 2018 to 1 December 2019.

Ethics and dissemination Ethical approval will not be required as our work is based on published studies. A list of all the studies included in this work will be available for review. We plan dissemination in a peerreviewed international scientific journal and through conference presentations. Our review will highlight the peer effect of peers in adolescent drinking behaviour and provide guidance for developing effective prevention and intervention programmes. We expect it to be informative for policy and practice, decision-making as well as

\section{Strengths and limitations of this study}

- Systematic review and meta-analysis of works that use stochastic actor-o riented models (SAOM) methodology to evaluate peer influence in adolescent drinking behaviour.

- The meta-analysis will offer a rigorous estimation of the magnitude of peer influence effect (free from the effects of social selection).

- Potential clarification as to whether peer influence in drinking behaviour differs based on the gender and age of the adolescents.

- The range of SAOM influence measures and structural effects used in different studies may inhibit the synthesis of outcomes.

- The majority of SAOM studies have been conducted in the USA and countries of Western Europe, which limits generalisation to other regions of the world.

for further research in public health and sociology of adolescents.

PROSPERO registration number CRD42019119836.

\section{INTRODUCTION}

Alcohol use from a young age constitutes a significant public health problem in many countries. Research shows that already by the age of $13,28 \%$ of teenagers will have tried alcohol; as they get older, the number of those who regularly drink alcohol increases as well as the quantity and variety of drinks consumed. ${ }^{2}$ Starting alcohol consumption early in life is harmful to the health of children and adolescents; it has a negative impact on physical and mental health in adulthood and it often leads to addiction. ${ }^{3-5}$

It is crucial to understand the factors associated with adolescent drinking in order to develop effective prevention programmes. ${ }^{6}$ Among the most consistent and important factors related to adolescent drinking are social influences. ${ }^{7-9}$ Peer relationships play a central role in an adolescent's life, comprising a social 
context for the development of a young person; children and teenagers are particularly susceptible to peer influence due to the utmost importance of peers at this developmental stage; moreover, the effects of peer influence are stronger during adolescence than in adulthood. ${ }^{10}$ Peer interactions may foster healthy or unhealthy development, including substance abuse. ${ }^{11-13}$ Some research shows that the tendency to be influenced by peers does not remain constant over time: it is higher during early and middle adolescence than during preadolescence or late adolescence. ${ }^{14-16}$ Other authors point out that these age patterns demonstrate gender differences and that this varies depending on the specific behaviour. ${ }^{17} 18$

Adolescents' alcohol consumption is closely associated with the drinking behaviour of their peers. ${ }^{9}$ It may thus be concluded that peer influence exerts a role in explaining the willingness to drink alcohol. Furthermore, there is a quantity of research studying different aspects of this process: peer norms, direct and indirect influences, popularity and friendship effects. ${ }^{19-21}$ However, the similarity in drinking behaviour patterns may arise from two different processes: the influence of peers on a person's behaviour or the preference of a person to associate with similar others. ${ }^{22}$ In order to evaluate peer influence, it is necessary to disentangle it from social homophily, or the tendency to affiliate with people with similar behaviour. ${ }^{23}$

We have been able to identify three systematic reviews of papers analysing the interrelations of adolescents' health risk behaviour and friendship networks. Jeon and Goodsonevaluated 15 articles based on the US representative dataset 'Add Health', which study the influence of friendship networks on adolescents' risk behaviour. ${ }^{24}$ Jacobs $e t$ al, reviewed the role of gender and network sex composition as a determinant of adolescents' risk behaviour. ${ }^{25}$ The papers included in their review encompassed a variety of methods: Exponential random graph models (ERGM), Quadratic assignment procedures (QAP), Agent based models $(\mathrm{ABM})$, regression, multivariate and bivariate statistics. A recent study from Leung $e t a l$ is a systematic review of 22 articles published between 1997 and 2011 which used longitudinal network studies intending to assess the effects of peer influence and selection process on adolescent alcohol use. ${ }^{26}$ Only a few reviewed papers used network design and network measures of friendship effects, while the majority were based on perceived measures of alcohol use or deviant behaviour. The described systematic review have used a variety of methods: Ordinary Least Squares Regression (OLS), logistic and hierarchical regressions, latent growth modelling, structural equation modelling, analysis of variance (ANOVA) and multivariate analysis of variance (MANOVA) ${ }^{24-26}$ None of these methods, however, are able to separate the effects of peer influence from the confounding effects of peer selection.

The analytic approach that allows separating selection and influence processes is stochastic actor-oriented models (SAOMs). SAOM is a statistical model for the simultaneous, mutually dependent, dynamics of a relation (or social network) of social actors and the behaviour of these actors. A social network may represent any type of social ties, for example, friendship; and behaviour may represent any changing actor's characteristic, for example, alcohol consumption. Actor-oriented models assume that social actors play a crucial role in changing their ties to other actors as well as in changing their behaviour. Thus, the process of co-evolution of networks and behaviour is regarded as a result of the actors' individual decisions and is modelled as a Markov process constructed from the smallest possible steps. SAOMs require longitudinal (panel) network data, that is repeated measures of network ties and actors' attributes. The actor-driven modelling approach can be applied for assessing the strength of both selection and influence processes simultaneously, controlling for the effects of each other. 222728

Since the inception of this methodology in 2001, about 200 papers studying co-evolution of network ties and different behaviours have been published. The possibility of performing a meta-analysis on SAOM papers has been demonstrated very recently by Gallupe $e t$ al, who analysed peer influence in offending behaviour. ${ }^{29}$

The range of health risk behaviour, including drinking, among adolescents, has been studied in the framework of longitudinal network design with subsequent SAOM analysis, ${ }^{23} 3031$ and it is worth noting that some studies pay special attention to age and gender differences in the effects of peer influence. ${ }^{32} 33$

The aim of the present study is to conduct a systematic review and meta-analysis of SAOM studies in order to examine the relative contribution and directionality of peer influence on alcohol consumption by adolescents. Taking into consideration that susceptibility of adolescents to peer influence depends on age and gender, ${ }^{14-18}$ these two variables are of special interest for our study.

\section{METHODS}

We have prepared the current protocol in accordance with the Preferred Reporting Items for Systematic Reviews and Meta-Analyses Protocols (PRISMA-P) guideline (online supplementary file 1). ${ }^{34}$ The start and end dates of the project of our systematic review and meta-analysis are 01 December 2018-01 December 2019.

\section{Study eligibility criteria}

To select the relevant works, we have set a number of procedural criteria of the studies' design and method, type of analysed behaviour, and participants:

1 . The study should conduct an analysis of longitudinal data using SAOM. The number of data collection waves is not a limitation provided that there are at least two waves.

2. The study should explicitly measure social ties between adolescents (friendship ties, romantic ties, support ties and so on).

3. The behaviour modelled in SAOMs should be alcohol consumption. Specifically, the outcomes may be any of the following: drinking quantity, drinking frequency, 
binge drinking, drunkenness, onset to first alcohol use, lifetime alcohol use.

4. The target population should be children and adolescents from 10 to 19 years old.

5 . The study manuscript should be available by September 2019 when the updated database searches will be conducted.

\section{Information sources and search strategy}

The current research collects and analyses studies published in academic journals, dissertations/theses, reports, conference materials. The articles will be searched in the following electronic databases: Web of Science, Scopus, PubMed, The Cochrane Library (Cochrane Database of Systematic Reviews, Cochrane Central Register of Controlled Trials (CENTRAL), EBSCOhost (MEDLINE, SocINDEX, Academic Source, ERIC), ProQuest (ProQuest Dissertations and Theses Global), PsycINFO (PsycNET), Excerpta Medica database (Embase) and Cumulative Index to Nursing and Allied Health Literature (CINAHL). The search will include titles and abstracts. We will use Google Scholar for control, but not as the main electronic database; rather, we will use it to check for unpublished studies and grey literature. This procedure will allow us to correct our search query if needed.

Search query will consist of three points that match the research question: drinking behaviour, SAOMs and adolescents, and will be formulated with the inclusion of the maximum number of synonyms corresponding to these three points. Previously conducted systematic reviews on similar topics will help to formulate accurate queries.

Point 1 'Drinking behaviour': drink* OR alcohol* OR booze OR tipple and other synonyms, as well as types of beverages-liquor OR wine OR beer, etc. We will also include 'Substance Abuse' to collect a wider selection of studies dedicated to drinking among adolescents.

Point 2 'Analytical approach': SAOM* OR 'stochastic actor-oriented' OR The algorithm Simulation Investigation for Empirical Network Analysis in R (RSIENA) OR 'stochastic actor-based' OR 'simulation investigation for empirical network' and other possible variations of the method name.

Point 3 'Adolescents': adolesc* OR youth OR 'young person' OR teen* OR student* OR 'peer group' OR child* OR pupil and other synonyms.

After the search query has been tested on Web of Science and Scopus, it will be adapted for other search engines. If the request is modified, another search will be conducted through all the electronic databases. All of the changes will be discussed by the research team.

After forming our database of articles, according to the inclusion criteria, the references will be cross-checked for possible relevant studies not found by the above search queries.

An example of a full electronic search strategy for the Web of Science is given in online supplementary file 2.

\section{Study selection process}

The selection process will be conducted by three members of the research team independent of each other.

First, after deleting duplicate articles from the lists retrieved from all electronic databases, three reviewers will make independent decisions on whether the article should be included based on its brief description. Each article will be classified as 'relevant', 'irrelevant' or 'unclear'. Articles classified as 'unclear' by at least one team member will be discussed by the research group. Articles classified as 'irrelevant' by two team members will be deleted.

In the second step, three reviewers will carefully examine the full texts of the remaining relevant articles, make independent decisions on the methodological quality of the studies and conclude whether the studies should be included in the meta-analysis. Disagreements about disputed cases will be resolved through general discussion.

\section{Data collection process}

The data collection form will be created and piloted through several iterations in the course of reviewing a sample of primary studies. When the data collection form is finalised, two reviewers will independently extract data from each eligible study, duplicating each other's work. Duplication is needed to reduce errors in data extraction. The third reviewer will compare the duplicate extractions, and all discrepancies will be resolved through general discussion. If multiple reports of the same study will be identified, only one extraction will be kept. Such identification can be done by comparing the records of these studies and finding identical project names, datasets, characteristics of the study design, samples or model specifications. In case of missing or incomplete information (ie, participants demographics or missing data treatment), authors of primary studies will be contacted (up to three email attempts per study).

\section{Data items}

The following data will be extracted from the selected articles:

1. Bibliometrics: authors, name, source, year of publication, language and country. Bibliometric data will be generated automatically and checked for consistency by the members of the research team.

2. Characteristics of the study: country(s) and year when the study was conducted, project, dataset, type of sample (general, high risk, other), sample size (number of participants and number of networks), gender composition of the sample, age of participants and socioeconomic status of participants.

3. Study design: number and frequency of survey waves, data collection techniques, name generator questions.

4. Description of 'alcohol drinking behaviour': drinking quantity, drinking frequency, binge drinking, drunkenness, onset to first alcohol use, lifetime alcohol use; scale assessing the frequency/intensity of alcohol consumption, the type of alcoholic beverage, etc.

5. Descriptions of covariates included in the model: gender, age, family socioeconomic status and so on. 
6. Description of participant dropout from wave to wave, description of missing data, treatment of missing data.

7. Model specifications.

8. Effect size data: parameter coefficients and SEs for selection effects, influence effects, behaviour dynamics effects, network structural effects.

\section{Primary outcome}

Estimation of the effect size of peer influence on alcohol consumption behaviour among adolescents of different age groups.

\section{Quality assessment and risk of bias}

Careful reviewing of available risk of bias assessment tools and quality assessment checklists revealed that none of them are applicable to non-experimental, longitudinal panel network studies. ${ }^{356}$ We do not expect high heterogeneity between studies as every eligible study should employ the same methodology; nevertheless, the important characteristics of study design and implementation may differ considerably. We devised a custom checklist of study characteristics to evaluate the quality of each study: the number of networks, the number of participants, response rate, attrition rate, the percentage of missing data, treatment of missing data, study limitations. The checklist will be pilot-tested with a few articles and modified before quality evaluation. When the checklist is finalised, two reviewers will independently evaluate each eligible study. If there is insufficient detail reported in the study, we will contact the primary study authors for more information (up to three email attempts per study). Disagreements will be resolved by general discussion.

While the sample size is important for statistical power, it is not a source of bias. Missing data, on the other hand, if not treated properly, would have a significant impact on research findings. One of the problems that emerge while working with longitudinal network data is the loss of a network agent due to attrition. Other reasons for missing data are item non-response and tie non-response. However, up to $10 \%$ of missing data does not lead to biased estimates as RSIENA package includes a data recovery procedure that replaces missing data using information from the previous or next wave or the global average. ${ }^{27}$ Though imputation reduces the risk of bias in evaluating effects, we will closely monitor the percentage of missing data reported in primary studies.

\section{Data synthesis}

If a sufficient number of studies using SAOM are found, we plan to extract model results from individual articles and to conduct a meta-analysis. A meta-analysis of SAOM results is straightforward because parameters coefficients are presented as log odds ratios and require no further conversion. However, in SAOM there are different effects for testing peer influence (average similarity, total similarity, average alter) that are not directly comparable. Hence, we will need reasonably similar models, which further narrows our base for meta-analysis.
Since for some age groups there might not be enough research available, we will need to merge some age categories of adolescents. We may need to consult child psychologists so as to make an informed decision on merging some of the age categories.

For assessing heterogeneity of individual studies, we will use tau squared calculated in metafor package. ${ }^{37} 38$ For meta-regression, we will use either robust variance estimation in robumeta package ${ }^{39}$ or multilevel model in metafor package. ${ }^{29} 38$

Post-hoc sensitivity analyses will be conducted to explore whether covariates (age, gender composition) or choice of outcome measure (specific drinking behaviour) could explain observed heterogeneity in results.

\section{Patient and public involvement}

Patients and/or the public are not involved in the current study.

\section{ETHICS AND DISSEMINATION}

The current study is based on secondary data and does not require ethical approval. A list of all the studies included in this work will be available for review.

This study will be the first systematic review and meta-analysis to focus on rigorous analysis of peer influence in adolescent drinking behaviour. We plan dissemination in a peer-reviewed international scientific journal and through conference presentations. We expect the results of our study to be informative for researchers of childhood and adolescence, public health researchers and practitioners. The results of the research will provide guidance for developing effective prevention and intervention programmes.

\section{Strengths and limitations}

The fundamental strength of the proposed systematic review/meta-analysis is the fact that it is based on articles using rigorous methodology allowing the separation of peer influence from peer selection in the range of adolescent alcohol consumption behaviour. 'Social contagion' with harmful habits has been a popular research topic, and many researchers have attempted to evaluate peer influence in health risk behaviour. Recently, several review articles summarise the findings in this area. ${ }^{24-26}$ However, the papers reviewed have used a variety of methods, none of which allows separation of the effects of peer influence from the confounding effects of peer selection.

Traditional tests of peer influence suffer from 'projection bias', that is, the tendency for people to overestimate the similarity between their behaviours and those of their peers and from the inherent entanglement of two processes: social influence and social selection. The only method (SAOM) able to isolate selection and influence was designed explicitly for this purpose less than 20 years ago. ${ }^{28}$

The SAOM method requires network data and longitudinal surveys, which are quite difficult to implement. Nevertheless, more and more papers employing this methodology are appearing. Papers using longitudinal network data and SAOM analysis will be collected and reviewed. 
A possible limitation is that not enough articles for meta-analysis will be found. Our inclusion criteria imply specific age of adolescents (10-19 years old). A second limitation is that the models for meta-analysis should be sufficiently similar. SAOM allows modelling of different peer effects (average similarity, total similarity and average alter) that may not yield similar interpretations. Moreover, structural effects included in the model may also differ; therefore, we will extract data for meta-analysis from studies that use comparable model specifications.

Contributors The study conception and design of the protocol is the result of the authors' combined work. VT and VI conceived the investigation and wrote the draft of the protocol. VI and DA edited the final manuscript and methodology of systematic review and meta-analysis.

Funding This work was supported by National Research University Higher School of Economics (project 'Socio-cognitive analysis of decision-making: modern models, analysis and application').

Competing interests None declared.

Patient consent for publication Not required.

Provenance and peer review Not commissioned; externally peer reviewed.

Open access This is an open access article distributed in accordance with the Creative Commons Attribution Non Commercial (CC BY-NC 4.0) license, which permits others to distribute, remix, adapt, build upon this work non-commercially, and license their derivative works on different terms, provided the original work is properly cited, appropriate credit is given, any changes made indicated, and the use is non-commercial. See: http://creativecommons.org/licenses/by-nc/4.0/.

\section{REFERENCES}

1. Inchley J, Currie D. Growing up unequal: gender and socioeconomic differences in young people's health and well-being. Health Behavior in School-aged Children (HBSC) study: international report from the, 2014. 2016 http://www.euro.who.int/_data/assets/pdf_file/0003/ 303438/HSBC-No.7-Growing-up-unequal-Full-Report.pdf?ua=1 (Accessed 18 Dec 2018).

2. Inchley J, Currie D, Vieno A, et al. Adolescent alcohol-related behaviors: trends and inequalities in the WHO European Region, 2002-2014. 2018 http://www.euro.who.int/ data/assets/pdf file/ 0007/382840/WH15-alcohol-report-eng.pdf?ua=1 (Accessed 18 Dec 2018).

3. de Winter AF, Visser L, Verhulst FC, et al. Longitudinal patterns and predictors of multiple health risk behaviors among adolescents: The TRAILS study. Prev Med 2016;84:76-82.

4. Patrick ME, Schulenberg JE. Prevalence and predictors of adolescent alcohol use and binge drinking in the United States. Alcohol Res 2014;35:193-200.

5. Mason WA, Toumbourou JW, Herrenkohl TI, et al. Early age alcohol use and later alcohol problems in adolescents: individual and peer mediators in a bi-national study. Psychol Addict Behav 2011;25:625-33.

6. Jaccard J, Levitz N. Parent-based interventions to reduce adolescent problem behaviors: New directions for self-regulation approaches. In: Oettinger G, Gollwitzer P, eds. Self-regulation in adolescence. New York: Cambridge University Press, 2015:357-88.

7. Clark AE, Lohéac Y. "It wasn't me, it was them!" social influence in risky behavior by adolescents. J Health Econ 2007;26:763-84.

8. Fisher LB, Miles IW, Austin SB, et al. Predictors of initiation of alcohol use among US adolescents: findings from a prospective cohort study. Arch Pediatr Adolesc Med 2007;161:959-66.

9. Simons-Morton B, Haynie DL, Crump AD, et al. Peer and parent influences on smoking and drinking among early adolescents. Health Educ Behav 2001;28:95-107.

10. Brown B. Adolescents' relationships with peers. In: Lerner R, Steinberg L, eds. Handbook of adolescent psychology. New York: Wiley, 2004:363-94.

11. Rubin KH, Bukowski WM, Laursen B. Handbook of peer interactions, relationships, and groups. The Guilford PressNew York, NY 2011;4.

12. Ali MM, Dwyer DS. Social network effects in alcohol consumption among adolescents. Addict Behav 2010;35:337-42.
13. Duncan SC, Duncan TE, Strycker LA. Alcohol use from ages 9 to 16: A cohort-sequential latent growth model. Drug Alcohol Depend 2006;81:71-81.

14. Berndt TJ. Developmental changes in conformity to peers and parents. Dev Psychol 1979;15:608-16.

15. Steinberg L, Monahan KC. Age differences in resistance to peer influence. Dev Psychol 2007;43:1531-43.

16. Sumter SR, Bokhorst CL, Steinberg L, et al. The developmental pattern of resistance to peer influence in adolescence: will the teenager ever be able to resist? J Adolesc 2009;32:1009-21.

17. Sim T, Koh S. A domain conceptualization of adolescent susceptibility to peer pressure. J Res Adolesc 2003:13:57-80.

18. Erickson KG, Crosnoe R, Dornbusch SM. A social process model of adolescent deviance: combining social control and differential association perspectives. J Youth Adolesc 2000;29:395-425.

19. Teunissen HA, Spijkerman R, Prinstein MJ, et al. Adolescents conformity to their peers' pro-alcohol and anti-alcohol norms: the power of popularity. Alcohol Clin Exp Res 2012;36:1257-67.

20. Bot SM, Engels RC, Knibbe RA, et al. Friend's drinking behaviour and adolescent alcohol consumption: the moderating role of friendship characteristics. Addict Behav 2005;30:929-47.

21. Deutsch AR, Steinley D, Slutske WS. The role of gender and friends' gender on peer socialization of adolescent drinking: a prospective multilevel social network analysis. $J$ Youth Adolesc 2014;43:1421-35.

22. Steglich C, Snijders TAB, Pearson M. 8. Dynamic networks and behavior: separating selection from influence. Sociol Methodol 2010;40:329-93.

23. Knecht AB, Burk WJ, Weesie J, et al. Friendship and alcohol use in early adolescence: a multilevel social network approach. Journal of Research on Adolescence 2011;21:475-87.

24. Jeon KC, Goodson P. US adolescents' friendship networks and health risk behaviors: a systematic review of studies using social network analysis and Add Health data. PeerJ 2015;3:e1052.

25. Jacobs W, Goodson P, Barry AE, et al. The Role of Gender in Adolescents' Social Networks and Alcohol, Tobacco, and Drug Use: A Systematic Review. J Sch Health 2016;86:322-33.

26. Leung RK, Toumbourou JW, Hemphill SA. The effect of peer influence and selection processes on adolescent alcohol use: a systematic review of longitudinal studies. Health Psychol Rev 2014;8:426-57.

27. Ripley RM, Snijders TAB, Boda Z, et al. Manual for RSiena. http:// www.stats.ox.ac.uk/ snijders/siena/RSiena_Manual.pdf (accessed 18 Dec 2018).

28. Snijders TAB. The statistical evaluation of social network dynamics. Sociol Methodol 2001;31:361-95.

29. Gallupe O, McLevey J, Selection BSJ. and Influence: A MetaAnalysis of the Association Between Peer and Personal Offending. $J$ Quant Criminol 2018;23.

30. Kiuru N, Burk WJ, Laursen B, et al. Pressure to drink but not to smoke: disentangling selection and socialization in adolescent peer networks and peer groups. J Adolesc 2010;33:801-12.

31. Osgood DW, Ragan DT, Wallace L, et al. Peers and the Emergence of Alcohol Use: Influence and Selection Processes in Adolescent Friendship Networks. J Res Adolesc 2013;23:500-12.

32. Burk WJ, van der Vorst $\mathrm{H}$, Kerr M, et al. Alcohol use and friendship dynamics: selection and socialization in early-, middle-, and lateadolescent peer networks. J Stud Alcohol Drugs 2012;73:89-98.

33. McMillan C, Felmlee D, Osgood DW. Peer Influence, Friend Selection, and Gender: How Network Processes Shape Adolescent Smoking, Drinking, And Delinquency. Soc Networks 2018;55:86-96.

34. Moher D, Shamseer L, Clarke M, et al. Preferred reporting items for systematic review and meta-analysis protocols (PRISMA-P) 2015 statement. Syst Rev 2015;4:1.

35. Sanderson S, Tatt ID, Higgins JP. Tools for assessing quality and susceptibility to bias in observational studies in epidemiology: a systematic review and annotated bibliography. Int J Epidemiol 2007;36:666-76.

36. Zeng X, Zhang Y, Kwong JS, et al. The methodological quality assessment tools for preclinical and clinical studies, systematic review and meta-analysis, and clinical practice guideline: a systematic review. J Evid Based Med 2015;8:2-10.

37. Higgins JP, Thompson SG. Quantifying heterogeneity in a metaanalysis. Stat Med 2002;21:1539-58.

38. Viechtbauer W. Conducting Meta-Analyses in $R$ with the metafor Package. J Stat Softw 2010;36:1-48.

39. Hedges LV, Tipton E, Johnson MC. Robust variance estimation in meta-regression with dependent effect size estimates. Res Synth Methods 2010;1:39-65. 